\title{
Conflicto y regulación. Acción pública y lógicas de mesogobierno en España: el caso de la aplicación de la LOGSE en Galicia
}

\section{Romain Pasquier *}

Después de cuarenta años de régimen franquista, el proceso autonómico inscrito en la Constitución de 1978 ha impulsado una importante y profunda política de regionalización en España y ha entrañado la creación de diecisiete Comunidades Autónomas. Sometido a fuertes tensiones políticas, este movimiento descentralizador condujo a una configuración de geometría variable donde los niveles de autonomía son diversos y evolutivos (Vallés y CuCHILlo, 1988). Si, por una parte, esta recomposición de las relaciones centro/periferia permite aliviar la urgencia de las reivindicaciones regionalistas, división política secular en España, produce también, a más largo plazo, transformaciones profundas de las modalidades de la acción pública.

En efecto, una de las principales transformaciones es la regionalización de una parte de la acción pública, pasando a ocupar una posición central en ésta los gobiernos y las Administraciones autonómicas. Eso se traduce en la formación, en España, de mesogobiernos o gobiernos de nivel intermedio, es decir, «conjuntos institucionales de posiciones de autoridad (y los aparatos administrativos correspondientes), cuya autoridad está respaldada y ratificada por el Estado, y cuya actividad está orientada hacia la satisfacción de intereses y de identidades sociales propios de los grupos subnacionales, funcionalmente o territorialmente diferenciados» (PÉrEz DíAz, 1994). La aparición de estos mesogobiernos plantea la cuestión de la coordinación de la acción pública entre los diferentes niveles de la Administración. Los gobiernos autonómicos no comparten con frecuencia ni los mismos intereses ni las mismas constricciones institucionales y políticas que la Administración central. Ellos deben conciliar sus intereses con los de los actores políticos e intereses territoriales. De este modo, las percepciones y representaciones de los «problemas» necesitan una intervención pública que puede variar según los territorios y los niveles administrativos. Estos principios nuevos hacen más compleja la acción pública y la transforman en procesos relativamente conflictivos (AGRANOFF, 1993; SuBIRATS, 1991).

El estudio de la aplicación de la LOGSE (Ley de Orientación General sobre la Educación) en la Comunidad Autónoma de Galicia de marzo a septiembre de $1996^{2}$ ayuda a aclarar ciertos aspectos de estas transformaciones. Esta Ley provocó un amplio conflicto social y político en Galicia. Se trataría, pues, de mostrar aquí cómo un conjunto de lógicas políticas regionales, en el centro de las cuales se encuentra el gobierno autónomo, modifican profundamente los objetivos iniciales de una ley nacional. Por eso nosotros trabajamos sobre la articulación entre policy y politics, es decir, en la integración en el análisis de la acción pública de un cierto número de variables sociopolíticas como, por ejemplo, la identidad y la configuración política del territorio regional, las estrategias diferenciadas de los actores políticos a escala regional y nacional, el contenido y las modalidades de intercambio políticos en el seno de las 
Comunidades Autónomas y/o con la Administración central. La consideración de estas dimensiones nos da una visión menos formal y más dinámica de las relaciones entre los distintos niveles de la Administración en España. Este artículo quiere ser una contribución a la comprensión de las nuevas realidades territoriales que se perfilan a escala europea (Muller, 1995).

Después de haber analizado los efectos de la estructuración de un espacio político gallego en la percepción de los «problemas regionales», veremos de qué manera los grupos sociales y políticos, cuya movilización está ligada a las características sociopolíticas singulares de esta región, desafian la definición de «interés regional» del gobierno autonómico. En fin, estudiaremos los intereses políticos que fuerzan a negociar al gobierno autonómico, así como los canales de regulación de este conflicto.

\section{Los efectos de la autonomía regional: la territorialización de los entramados políticos}

En su análisis de la entrada en la agenda política de un problema, P. GARRAUD (1990) apunta que falta prestar atención: «al papel de la estructura de juego y de la competición política así como al peso de la coyuntura económica o ideológica que puede impulsar una sensibilidad particular a ciertos tipos de problemas (...)». En Galicia, la autonomía regional ha reforzado un sentimiento identitario preexistente y ha contribuido a la estructuración de un espacio de competición política con reglas de juego adaptadas a los intereses territoriales. Esta evolución sensibiliza de manera creciente a los actores políticos y sociales gallegos en la defensa de los intereses y peculiaridades propias de Galicia. Éste es el marco que es necesario entender para comprender las movilizaciones contra la forma específica de implantación de la LOGSE en Galicia.

\section{Galicia, una «nacionalidad histórica»}

Una unificación territorial tardía sumada a un proceso inacabado de centralización explica la permanencia de identidades regionales y el desarrollo de nacionalismos periféricos en España (LiNZ, 1973). Galicia es una de las tres «nacionalidades históricas» que reconoce la Constitución de 1978. Sin embargo, su trayectoria social, histórica y política la diferencian netamente de Cataluña y el País Vasco. Para Stein RokKan, «Galicia es un caso intermedio entre las periferias victoriosas y las perdedoras» (Díaz LOPEZ, 1982). Es victoriosa en el sentido de que ella fue capaz de conservar una cultura propia a lo largo de los siglos, pero es perdedora en la medida en que los movimientos nacionalistas influyen sólo de forma marginal sobre el centro, a diferencia de lo que ocurre en los casos catalán y vasco.

El aislamiento geográfico de Galicia en la Península Ibérica, así como su inferior desarrollo económico han facilitado la permanencia de estructuras sociales tradicionales y de una cultura popular particular, distinta de la del resto de España. La lengua vernácula, el gallego, encarna bien la especificidad cultural de esta región. La exaltación de esta especificidad sociocultural por parte de reconocidos intelectuales del siglo XIX y de comienzos del siglo xx produjo el despertar de la toma de conciencia de la existencia de una identidad diferencial en Galicia y, después de la primera guerra mundial nace el primer movimiento político nacionalista. Pero esta sociedad no ha producido nunca las precondiciones sociales y politicas requeridas para la organización de partidos nacionalistas de masas (Márz, 1996). Ni en el pasado, ni durante la transición democrática, el nacionalismo gallego ha igualado los niveles de implantación social, de presencia política y de apoyo electoral de los náacionalismos vasco y catalán. Por lo tanto, la «gestión franquista del territorio», caracterizada por una centralización forzada y una oposición categórica a toda forma de regionalismo (GARRAUD, 1994), no alteró las particularidades sociales y culturales gallegas. Desde entonces, la reactivación de este punto conflictivo socioelectoral durante la transición democrática por los partidos nacionalistas y los partidos de izquierda (PSOE y PCE) permitió a Galicia obtener, en 1981, un Estatuto de Autonomía comparable al vasco y al catalán.

\section{La estructuración de un espacio político regional}

Una de las principales consecuencias políticas de la autonomía regional en España es la formación de espacios regionales de competición política estructurados alrededor de áreas de intereses específicas. Los grados de autonomía de estos espacios políticos regionales son variables según las Comunidades Autónomas. En Galicia, además de la existencia de una fuerte identidad regional, existe un conjunto de dinámicas políticas que han contribuido a una fuerte autonomización del espacio político regional. De este modo, las elecciones autonómicas aprecian un descenso claro de las tasas de abstención ${ }^{3}$ (VILAS NogueIRA, 1992). Esto traduce la politización creciente de esta comunidad y la progresión de su sentimiento identitario. Es más, se asiste en Galicia, al igual que Cataluña o Andalucía, a la emergencia de elites políticas intermedias que se distinguen de las elites políticas locales por sus características sociales, así como por la naturaleza de sus propios recursos políticos ${ }^{4}$. 
Es más, el acceso limitado de las elites gallegas intermedias al centro político ha reforzado el interés de la competición política regional. Esta situación es la resultante de la configuración política gallega durante el período de dominación del PSOE sobre la vida política española. En efecto, en el seno de las instancias nacionales del PSOE, el Partido Socialista de Galicia (PsdeG-PSOE) permaneció bastante marginado. Durante los catorce años de gobierno González, no obtuvo más que un solo cargo ministerial en Madrid, el Ministerio de Transportes. En comparación con otras Comunidades Autónomas como Andalucía, el acceso de los dirigentes socialistas gallegos a puestos de dirección política fue muy restringido. Por otra parte, la oposición política entre un gobierno central dirigido por los socialistas y un gobierno autonómico ocupado por la derecha gallega ${ }^{5}$ amplifica la marginalización política de Galicia en los años 80. Es solamente en el marco de las instancias nacionales del Partido Popular (PP) y en la influencia de Manuel Fraga donde los actores politicos que dirigen la Comunidad Autónoma tienen acceso al centro político. Estos factores de aislamiento político, que en el artículo merecerán un estudio más profundo, tienen como consecuencia una regionalización creciente de los intereses políticos. Sin embargo, la victoria del PP en las elecciones generales de marzo de 1996 y la entrada en el gobierno Aznar de numerosos miembros del Partido Popular de Galicia (PPdeG) ${ }^{6}$ marca el inicio de una «estrategia de integración vertical» (GENIEYS, 1996) del centro político por las elites intermedias gallegas.

La competencia entre los partidos políticos en el espacio político regional produjo igualmente una territorialización de las estrategias y un cambio en el registro discursivo por parte de los actores políticos. De este modo, el derribo en 1987 del gobierno conservador por una coalición parlamentaria compuesta por el PSOE y dos partidos nacionalistas moderados, Coalición Galega y Partido Nacionalista Galego, está en el origen de un cambio de estrategia de la derecha gallega. Gracias al impulso de Manuel Fraga, el PPdeG adopta en adelante un contenido resueltamente regionalista que se distingue del discurso, tradicionalmente centralista de la derecha española. Para conservar su ventaja política y contrarrestar la progresión del voto nacionalista ${ }^{7}$, el PPdeG, afianzado en la sociedad rural gallega, hace suyos sin apenas dificultad los elementos culturales gallegos, especialmente la lengua. De este modo, Manuel Fraga, en el curso de sus intervenciones evoca regularmente a los pioneros del «galleguismo político» y estigmatiza la «deuda histórica» de España con Galicia en cuestión de carreteras, de desarrollo económico, etc. Esta estrategia del PPdeG así como la oposición política de la Xunta al gobierno central convirtieron a Galicia en una de las Comunidades Autónomas más reivindicativas en términos de transferencias de competencias y de conquista de nuevos recursos financieros durante los mandatos de Felipe González. La Xunta aparece así ante la opinión pública como la principal defensora de los intereses de Galicia tanto frente al Estado Central como frente a las otras Comunidades Autónomas y a las directivas de la Comisión Europea.

Esta situación produce en reciprocidad ajustes en el seno de los otros partidos políticos. El PSdeG reclama igualmente su «galleguismo» y pretende defender las especificidades sociales, económicas y culturales de Galicia ${ }^{8}$. Algunos de sus miembros desean incluso que se emancipe de la posición de las instancias nacionales sobre la cuestión nacional a semejanza del Partido socialista catalán. En cuanto al BNG, partido nacionalista de izquierdas, reforzado tras los últimos procesos electorales, denuncia por su parte el regionalismo tímido del PPdeG y del PsdeG y asegura que estos dos partidos no defienden los intereses gallegos de la misma forma que lo hace CiU en Cataluña o el PNV en el País Vasco. De esta forma, cada uno de los partícipes del juego político regional pretende ser el representante auténtico de los intereses de Galicia y el defensor de su particularidad.

La estructuración del espacio político gallego en torno a cuestiones territoriales no implica que este espacio esté cerrado herméticamente a las grandes cuestiones de interés nacional. Al contrario, el conjunto de los partidos políticos, ya sean éstos nacionalistas o de carácter estatal, participan activamente en el juego político nacional. Diversas redes políticas, especialmente las redes partidistas, articulan el espacio político regional con el escalón nacional y los hace interdependientes. Por otra parte, el marco institucional del Estado de las Autonomías limita el papel de la Xunta, en diferentes ámbitos de acción pública, a aplicar las directivas de la Administración central. No obstante, la institucionalización de las autonomías modifica la conciencia regional, así como la percepción de los problemas situados a este nivel (Botella, 1989). La estructuración, en Galicia, de un espacio político regional en torno a las cuestiones propias de la Comunidad sitúa en un lugar central del debate la cuestión del «interés regional». Es en el nombre del «interés regional» que los diversos grupos sociales y políticos van a contestar el modo de implantación de las directivas emanadas de la LOGSE por parte de la Xunta de Galicia.

\section{La lógica del mesogobierno con relación a las movilizaciones infrarregionales}

\section{La génesis de un conflicto}

En principio, esta reforma educativa se sitúa en el marco 
de lo que se puede considerar federalismo de ejecución (Mo. RATA, 1993). En materia de educación, la Constitución española atribuye al nivel superior la edición de las normas de base, mientras que las Comunidades Autónomas son beneficiarias de transferencias de competencias que aseguran la ejecución administrativa de la legislación estatal. De este modo, la Comunidad Autónoma de Galicia, de acuerdo con las competencias transferidas en materia de educación, ha recibido el encargo de poner en práctica las disposiciones de la LOGSE, Ley que reemplaza a la Ley General de Educación de 1970. La LOGSE instaura el carácter obligatorio de la escolarización hasta los 16 años y prevé la división del período educativo en dos etapas. Una primaria o elemental, de seis a once años, y una nueva etapa denominada ESO ${ }^{9}$ (Enseñanza Superior Obligatoria) de doce a dieciséis años. La Ley fija igualmente el calendario detallado de su aplicación a partir del comienzo del curso 1996-1997. Para poner en práctica las disposiciones recogidas en la LOG$\mathrm{SE}$, la Consellería de Educación de la Xunta ${ }^{10}$ elaboró, durante el año 1995, la red de centros que impartirán los cursos de la ESO, dotándolos de este modo del personal y el material adecuado.

La elaboración de esta red de centros no se realizó con perfecta transparencia. La Consellería de Educación difundió una información mínima sobre la elaboración de su proyecto durante el año 1995, y retardó la publicación de la versión definitiva del mapa de centros, inicialmente prevista para comienzos del año 1996. Las circunstancias políticas nacionales explican el comportamiento del departamento de educación y, de manera más general, el de la Xunta. A comienzo del año, nos encontramos con un contexto político nacional muy particular. En efecto, en las elecciones de marzo de 1996 el PP está en condiciones de obtener la mayoría en las Cortes por primera vez desde la transición democrática. Ésta es una oportunidad política única para el PP. La Xunta y su presidente, Manuel Fraga, no quieren arriesgar el éxito electoral del PP publicando antes del 3 de marzo el mapa de centros. En efecto, la estrechez de los márgenes presupuestarios de la Comunidad Autónoma de Galicia ${ }^{11}$ obligó a la Consellería de Educación a racionalizar fuertemente el número de centros y su dotación de material y personal, especialmente en las zonas rurales. El proyecto suscitaba, en Galicia, las inquietudes de asociaciones de padres de alumnos, de alcaldes, así como las protestas de los sindicatos de profesores.

El mapa de centros no fue publicado hasta unos días después de las elecciones generales. Éste divide a Galicia en 108 zonas escolares y prevé la puesta en práctica transitoria de la LOGSE en un período de cuatro años. De este modo, al comienzo del curso 1996-1997, la Consellería de Educación preveía que el $68 \%$ de los alumnos de colegios públicos que comenzasen el primer curso de la ESO permaneciesen en su centro de origen. Este período transitorio debería permitir a la Xunta hacer las inversiones necesarias para construir los nuevos centros de la ESO y adaptar los medios materiales y humanos. Sin embargo, a pesar de este período transitorio, la consecuencia inmediata de la implantación de esta red es el desplazamiento forzado al comienzo del curso 1996-1997 de varios millares de alumnos (aproximadamente tres mil). Estos desplazamientos de alumnos, a veces a decenas de kilómetros, afectan especialmente a las pequeñas comunidades rurales de las provincias orientales de Galicia ${ }^{12}$, en las cuales la red no previó la instalación de un centro de ESO debido al reducido número de alumnos. Éstos son obligados a frecuentar los centros de ESO limítrofes. La constancia de este hecho provocó las reacciones inmediatas de los padres de los alumnos y de los alcaldes afectados durante los primeros días del mes de marzo.

\section{La dinámica histórica del territorio: localismo y caciquismo}

Las primeras movilizaciones contra la aplicación de la ESO se desarrollaron en ciertas comunidades rurales de la provincia de Lugo. Los alcaldes y las asociaciones de padres de alumnos de estas comunidades no aceptaron el traslado de sus hijos a los centros limitrofes. Estas protestas fueron, tanto en la forma como en la localización, fuertemente localizadas ${ }^{13}$. Fueron asociadas a la estructuración social y política de estos territorios, caracterizados por la permanencia de espacios locales, lugares de memoria y de formación de identidades y de prácticas polí. ticas especificas (BRIQuet y SAVICKI, 1989).

J. Linz (1966) subraya en un artículo seminal la heterogeneidad económica y social de las regiones españolas y la diversidad consiguiente en el reclutamiento de las elites. Hasta los años 60-70 Galicia permaneció como una sociedad fundamentalmente agraria y subdesarrollada económicamente. La actividad agricola estaba orientada hacia la autosubsistencia (policultivo) y se apoyaba sobre un sistema de pequeñas explotaciones de carácter familiar denominado minifundio (ITURRA, 1988). La extrema partición de las tierras y una geografía accidentada producían un hábitat muy disperso, compuesto de multitud de aldeas. Los medios de comunicación, casi inexistentes, limitaban el intercambio económico a los espacios próximos. Las comunidades rurales están divididas en múltiples parroquias ${ }^{14}$. Éstas forman unidades geográficas, sociales y culturales bien delimitadas que constituyen el marco de referencia inmediato para la población (Lisón TOLOSANA, 1974). La parte esencial de la vida cotidiana se desarrolla en el interior de la parroquia. Veinte años después, Galicia ha conocido profundos cam. bios económicos y sociales que modifican considerablemente este esquema. Mas estas transformaciones no afectan a todas las partes del territorio al mismo ritmo. De este modo, una diferenciación, cada vez más nítida, se puede hacer entre una Galicia occidental, cada vez más urbanizada y una Galicia orien- 
tal que conserva aún los rasgos característicos de una sociedad rural.

La división geográfica, económica y social de Galicia se refuerza con el control eclesiástico de las parroquias y con un modo clientelar de ejercicio del poder político, el caciquismo. El caciquismo designa una forma clientelar de dominación política, sistematizada por el régimen de la Restauración ${ }^{15}$. Pero el caciquismo perduró más allá de este período, no en vano una de sus características principales es la adaptabilidad: los regímenes y los partidos políticos cambian, pero el cacique y su clientela permanecen. De este modo, a través de los distintos regímenes políticos españoles del siglo $\mathrm{xx}$ y aún hoy en día, este tipo de dominación política permanece vivo en ciertas regiones españolas, particularmente en Galicia. J. F. MEDARD (1976) define la relación de clientela como «una relación de dependencia personal no vinculada a relaciones de parentesco, que descansa sobre un intercambio recíproco entre dos personas, el patrón y el cliente, que controlan recursos desiguales [...]». Se puede completar esta definición subrayando que la relación patrón-cliente funciona con un fuerte contenido simbólico, es decir, con gratitud y respeto (ROMERo MAURA, 1977). De esta forma, en Galicia, sobre un territorio disperso, el cacique dispone de recursos sociales suficientes, principalmente de relaciones sociales extracomunitarias, para intervenir frente a una u otra Administración o autoridad con el fin de prestar un servicio a un individuo o un grupo de individuos. A cambio, el cacique puede contar con el apoyo electoral del patrocinado que ha obtenido el favor. La organización reticular del caciquismo le presta operatividad. En efecto, el cacique de la comunidad, que normalmente acostumbra a ser el alcalde, cuenta con enlaces en cada parroquia o unidad de población. Este enlace asegura un contacto directo con una población dispersa, marcada por fuertes identidades parroquiales, y permiten el ascenso de las demandas de los administrados. El cacique comunal se inscribe el mismo en una relación jerárquica con el cacique de la provincia que dispone de relaciones con los representantes del Estado. Si el cacique de una determinada comunidad no puede resolver un problema, demanda entonces la intervención del cacique provincial que juega el papel de intermediario con la Administración central.

La evolución económica y social de la sociedad gallega, la autonomía regional y la politización creciente de los habitantes han transformado este tipo de dominación política. Sin embargo, numerosos indicadores permiten pensar que las relaciones políticas clientelares se han adaptado a la autonomía y a la regionalización de la vida política en Galicia. Estas prácticas, que se inscriben en una relación singular con lo político, contribuyen en buena medida a través de la intermediación de las instancias de representación y de mecanismos de legitimación y de acción política al funcionamiento concreto de las instituciones regionales (BRIQUET, 1995). De este modo, desde el período de la transición, Alianza Popular, que dispone de sólidos apoyos en Galicia, se constituye a partir «de una vaga federación de notables, cada uno de los cuales poseedor de su propia base - clientelista y/o geográfica - caracterizada por una débil organización, una ausencia casi total de programa y de ideología (...) y apoyada sobre relaciones interpersonales jerárquicas y de tipo feudal» (GUNTHER et al., 1986). Si la organización partidaria de la derecha española se refuerza grandemente con estas prácticas, la estructuración social y política de Galicia facilita a su vez la permanencia de prácticas políticas clientelares en el seno del PPdeG ${ }^{16}$ (Cazorla Pérez, 1994). La continuidad de las elites políticas locales en Galicia entre los últimos años del período franquista y el período contemporáneo constituye un indicador suplementario de la permanencia de este tipo de prácticas, en particular en las zonas rurales ${ }^{17}$. La historia sociopolítica de la región facilita así la reproducción de fuertes identidades locales y de un «poder territorial» en las provincias (VILAS NogueIRA et al., 1994).

La lógica de esta singular relación política permite comprender la implicación de los alcaldes en el conflicto de la ESO. Los actores sociales implicados por la aplicación del proyecto de la Consellería de Educación forman parte potencial de las clientelas de estos alcaldes. La falta de reacción por su parte significaría la pérdida de apoyos electorales. De este modo, ellos demandaron de su «patrón», uno de los líderes del PPdeG, que hiciese presión, conjuntamente con ellos, sobre el gobierno autónomo.

\section{Redes políticas clientelares y defensa de las especificidades de la Galicia rural}

Las dinámicas del espacio político regional transformaron un conflicto, en su origen muy localizado, en un conflicto político de envergadura. En efecto, el sostén de las redes políticas clientelares del PPdeG a los afectados por la ESO les permitió a éstos ejercer una fuerte presión política sobre la Consellería de Educación para que renunciase al mapa de centros que había diseñado. Se entabló, por lo tanto, una lucha entre la Xunta y los grupos movilizados por la definición del «interés regional».

El «interés regional» que la Xunta pretende defender a través de este plan es contestado por diferentes grupos sociales y políticos. La Consellería de Educación argumenta que la ESO, al racionalizar los medios humanos y financieros, aumentará la calidad de la enseñanza en Galicia. Los actores sociales y políticos que se movilizan tienen, por su parte, otra visión del «interés regional». Ellos subrayan las especificidades de la Galicia rural, como, por ejemplo, la extrema dispersión de la pobla. ción y estiman que la Consellería debería respetar e integrar estas características en su proyecto. Los primeros en manifestarse fueron los alcaldes de los municipios rurales de la provincia 
de Lugo. En efecto, los padres de los alumnos que protestan contra el proyecto de la Xunta representan la clientela de estos alcaldes. Una ausencia de reacción significaría el cuestionamiento de la «confianza» de los administrados sobre la capacidad del alcalde de defender los intereses de la comunidad y de resolver sus problemas. Por lo mismo, la marginalización demográfica y económica de estas comunidades se traduciría en la pérdida de recursos sociales y políticos para el alcalde.

En estas condiciones, los alcaldes del PPdeG de la provincia de Lugo se volvieron hacia su «patrón» el presidente de la Diputación provincial y presidente provincial del PP, Francisco Cacharro Pardo. El capital político del presidente de la Diputación emana directamente del apoyo que le prestan las redes políticas locales controladas por los alcaldes, pues los diputados provinciales son elegidos según un modo indirecto de elección sobre la base de los resultados obtenidos por los diferentes partidos en las elecciones municipales. La Ley electoral prevé la elección de los diputados provinciales entre los concejales elegidos en el marco de los partidos judiciales. Estas redes, sistemas de afinidades duraderas fundadas sobre relaciones de dependencia y de obligación (SAWICKI, 1988), recubren las prácticas políticas que funcionan según una lógica clientela organizadas a nivel provincial. En efecto, si la provincia como escalón de la Administración territorial se debilitó poco después de la creación de la Comunidad Autónoma ${ }^{18}$, ella permanece, sin embargo, como un espacio determinante de organización en la política en Galicia. La provincia constituye la circunscripción de base para las elecciones generales y autonómicas. El PP se estructura a partir de este escalón. Todas las quejas de los alcaldes suben hacia su «patrón», el presidente de la Diputación. Éste, por consiguiente, uno de los líderes del PP gallego, tomó partido por los alcaldes presentándose como un intermediario entre los grupos movilizados y la Xunta. Entre la pérdida de sus apoyos electorales y el sostén de su partido en el gobierno regional, el presidente de la Diputación de Lugo no dudó y presionó para la flexibilización de la reforma de la ESO.

Los alcaldes rurales del PPdeG utilizaron su peso electoral para hacer ceder a la Xunta. El PPdeG retiene una abrumadora mayoría de las alcaldías de la Galicia rural. En las elecciones locales de 1995 el 83,6\% de las alcaldías de Lugo pertenecieron al Partido Popular ${ }^{19}$. De este modo, el 12 de marzo de 1996 treinta alcaldes del PPdeG de la provincia de Lugo se reunieron con el conselleiro de Educación, Piñeiro Permuy, en los locales. del PP para demandar rectificaciones a la aplicación de la LOG. SE. Los alcaldes acusaron a la Xunta de marginalidad a las comunidades rurales. De esta forma, el alcalde de Baleiras amenazó con «consecuencias desagradables si el gobierno gallego no cedía» y añadió que él sería «el primero en tomar la cabeza de las movilizaciones contra lo que él considera como una marginación de su comunidad» y que «Xoán Piñeiro Permuy des- conocía a su comunidad y al resto de la provincia de Lugo». Las amenazas con respecto a la Xunta y su Conselleiro de Educación se hicieron más y más precisas. El 26 de marzo los alcaldes de Samos, O Incio y Paradela indicaron que «las dimisiones se sucederían en el partido si el programa de la red de centros se mantenía» ${ }^{20}$. Éstos precisaron que la Consellería de Educación no puede aplicar la LOGSE de la misma forma en toda Galicia y demandaron la dimisión del Conselleiro. Al mismo tiempo la Federación Galega de Municipios e Provincias (FE. GAMP) y la Federación de comunidades gallegas pidieron a la Consellería que atendiera las reinvindicaciones de los alcaldes ${ }^{21}$.

Esta movilización de los intereses políticos locales a escala provincial produjo un fuerte impacto político y dio importancia a las reivindicaciones. Las protestas se extendieron rápidamente a gran parte de Galicia. A finales de marzo la reforma de la ESO se transformó en un conflicto político a gran escala. Todas las provincias son afectadas. Las asociaciones de padres de alumnos de los municipios afectados se movilizaron y multiplicaron sus acciones: manifestaciones, huelgas, ocupaciones de locales, cierres de comercios, peticiones, cortes de tráfico y repetidas demandas de entrevistas con el Conselleiro de Educación. Estos actos de protesta se realizaron de una forma dispersa hasta la creación, a comienzos de abril, de una coordinadora formada por una docena de personas, encargada de dirigir y programar las movilizaciones. La presencia de numerosos alcaldes del PP en la creación de esta coordinadora dota a ésta de una legitimidad política inmediata. Desde este momento, las modalidades de acción y los modos de expresión de las reivindicaciones de los «afectados» se organizan a escala política regional. El 19 de abril miles de personas venidas de todas las provincias de Galicia se manifestaron en las calles de Lugo. El 9 de mayo se secunda, de forma masiva, una huelga general de colegios de educación secundaria en la provincia de Lugo. La coordinadora utiliza el argumento de la especificidad, enfatizando en sus reivindicaciones las singulares características socio-económicas de Galicia, para criticar el mapa de centros elaborado por la Consellería de Educación. Esta coordinadora exige, en fin, un conjunto de medidas por parte de la Xunta: una moratoria en la aplicación del plan de la Xunta que incluya los dos siguientes cursos escolares; congelar la aprobación de los proyectos de centro; apertura inmediata de un período de debate con los afectados, así como demandar la existencia de servicios educativos obligatorios públicos encaminados a mantener la población y las actividades económicas, sociales y cul. turales en los medios rurales ${ }^{22}$.

El conflicto entre la Xunta y estos grupos produjo efectos inmediatos en el espacio político autonómico. El conjunto de los actores políticos autonómicos se posicionó con relación a este problema. Se asiste, en este caso, a una regionalización del debate. Los portavoces de educación de los distintos grupos 
parlamentarios interpelaron al Conselleiro de Educación bien a través de los medios de comunicación, bien por medio de preguntas en el Parlamento autonómico. El BNG se pronunció a favor de una congelación provisional del proyecto con la fina. lidad de adaptar la LOGSE a las realidades geográficas de Galicia y mantener, por tanto, una enseñanza pública vertebrada del territorio. La posición del PSdeG es más ambigua. Defiende los principios de la LOGSE, elaborada por un gobierno socialista, pero se desmarca a su vez de la forma en que la Xunta la aplica. Para el PSdG, la ausencia de negociaciones a la hora de elaborar la red de centros entre la Consellería de Educación y las asociaciones de padres de alumnos, los sindicatos de profesores y los grupos parlamentarios ha pervertido los objetivos de la LOGSE. Por el contrario, la izquierda gallega (EU-EG) se pronunció a favor del mapa de centros ${ }^{23}$. Los sindicatos CCOO y FETE/UGT adoptaron la misma posición aunque demandando ciertas adaptaciones en atención a la realidad gallega.

Enfrentado a la multiplicación de los actos de protesta, el Conselleiro de Educación intentó en numerosas ocasiones apaciguar las movilizaciones precisando el carácter obligatorio de la aplicación de la LOGSE, Ley perjudicial en su opinión, pero hecha aceptable y operacionalizable gracias a la red de centros elaborada por su departamento. Convocó un pleno del Parlamento autonómico para explicar su proyecto ante la oposición parlamentaria e inició el diálogo con los protagonistas del conflicto. El proyecto del Conselleiro recibió el apoyo del Presidente de la Xunta, Manuel Fraga. Sin embargo, si el departamento de Educación acepta discutir algunas cuestiones marginales del proyecto, principalmente las que afectan a las localidades de alta montaña, por otra parte, rechaza las exigencias de los alcaldes y las negociaciones directas con la coordinadora de afectados. Desde entonces, el departamento de Educación y su Conselleiro se encuentran sin relación con las redes políticas locales, organizadas por el presidente de la Diputación de Lugo, que negocian directamente en el marco del PPdeG una modificación de las modalidades de implantación de la LOGSE.

\section{Lógicas partidistas y regulación política del conflicto}

De este modo, para retomar la distinción conceptual de B. Jobert (1995) aplicada a las policy communities, la Comunidad Autónoma de Galicia terminó por constituirse como un foro y una arena regionales de política pública, es decir, a la vez un lugar de debate y de negociación. Durante el curso de este largo proceso, a lo largo del cual se construyó progresivamente un compromiso político, se advierte toda la importancia de la red partidista del PP, la cual facilita la articulación entre los diferentes actores y los distintos niveles de Administración implicados en el conflicto de la ESO.

\section{Disensiones en el seno del PPdeG}

Organizado sobre una base provincial, el PPdeG se compone de varias organizaciones políticas que integran a nivel provincial múltiples redes políticas locales. Esta estructuración del PPdeG produce numerosas tensiones entre sus diferentes organizaciones provinciales. Esta imbricación de redes políticas locales en el seno del PPdeG limita de forma considerable los márgenes de maniobra de la Xunta. En efecto, son estas relaciones de competencia en el seno del PPdeG las que empujan a ciertos actores de este partido a hacer presión sobre el gobierno autonómico para que flexibilice la puesta en funcionamiento de la LOGSE en el sentido propugnado por los alcaldes y la coordinadora de afectados. De este modo, el conflicto de la ESO se tradujo en enfrentamientos internos dentro del PPdeG.

La coordinadora, los alcaldes rurales y el Presidente de la Diputación de Lugo encontraron aliados en el secretario general del PPdeG, José Cuiña Crespo, y en los cargos electos que le apoyan (alcaldes y diputados autonómicos). Las movilizaciones de los agentes sociales y políticos opuestos a las formas de aplicación de la ESO sacan a la luz del día las numerosas disputas internas entre las distintas sensibilidades del PPdeG. Estas luchas tienen un catalizador principal: la sucesión de Manuel Fraga en la Presidencia de la Comunidad Autónoma y la consiguiente redistribución de puestos de poder. Para el Presidente de la Diputación de Lugo, y presidente provincial del PP, el conflicto de la ESO es la ocasión de conseguir nuevas parcelas de poder en el seno del PPdeG en un momento en el que se están redistribuyendo las cartas del juego político regional. Cacharro encuentra un aliado en la figura de José Cuiña, secretario general del PPdeG y Conselleiro de Ordenación del Territorio y Obras Públicas de la Xunta de Galicia. Éste aparenta postularse como pretendiente a obtener la sucesión del Presidente de la Xunta, apoyado sobre los recursos políticos de los actores políticos territorializados. Antiguo alcalde de un municipio rural y antiguo presidente de la Diputación Provincial de Pontevedra, ha fundamentado su carrera política en el control de las redes politicas locales de tipo clientelar. En el interior del PPdeG, el sector de José Cuiña tiene el sostén de los presidentes del PPdeG de las Diputaciones provinciales de Galicia, ellos mismos apoyados por los alcaldes del PP. Sobre la base de estos recursos políticos territoriales, este sector político defiende la estrategia de galleguización del discurso del PPdeG. Este sector se opone a otros grupos dentro del PPdeG, que acumulan recursos procedentes de las instancias nacionales 
del PP, y que buscan a la vez acceder a puestos en el poder central y en la Xunta de Galicia.

Las redes políticas locales movilizadas contra la aplicación de la ESO en Galicia obtuvieron, por consiguiente, el apoyo del secretario general del PPdeG, pues constituyen apoyos políticos fundamentales para él en esta situación de fuerte competencia política. Mientras que numerosos alcaldes continuaban amenazando con abandonar el PPdeG si la Xunta no renunciaba a su proyecto, el secretario general del PPdeG se presentó como mediador entre los alcaldes del medio rural, por un lado, y el Presidente de la Xunta y su Conselleiro de Educación, por el otro. De este modo, él se reunió en numerosas ocasiones con delegaciones de alcaldes a finales de abril. El secretario general del PPdeG, miembro influyente del gobierno autonómico, hizo entonces presión sobre el Presidente de la Xunta, para que modificase su posición respecto de las directrices de implantación de la ESO elaboradas en la Consellería de Educación.

En esta relación de fuerza entre las redes locales y la Xunta, hizo resaltar la importancia del voto rural en Galicia. En efecto, el sistema electoral en vigor para las elecciones autonómicas sobrerrepresenta al voto rural. La relación entre número de escaños y número de electores es mucho menos elevada en las provincias orientales de Galicia, mucho más ruralizadas y con menor población que las provincias occidentales ${ }^{24}$. Si se añade a este factor el voto tradicionalmente conservador de la Galicia oriental y la convocatoria en octubre de 1997 de elecciones autonómicas, se comprende mejor la magnitud de la presión ejercida por la coordinadora y los alcaldes del PPdeG sobre la Xunta y su presidente.

\section{Redes partidistas y negociaciones transversales}

Estas presiones políticas y la multiplicación de las protestas obligaron al gobierno autonómico a negociar para evitar un rechazo total de la reforma de la ESO. La toma de posesión, a comienzos de mayo, del gobierno de José María Aznar es un dato importante para comprender los procesos de negociación. En efecto, la conquista del poder estatal por el PP permitió a la Xunta implicar a la Administración central en la resolución del conflicto y apaciguar al mismo tiempo las tensiones en el espacio político regional. Es entonces en el marco interno de relaciones del partido popular donde se van a establecer las negociaciones transversales ${ }^{25}$ entre los grupos sociales y políticos movilizados contra la aplicación de la ESO, el gobierno autonómico y el Ministerio de Educación. De este modo, en el momento de la negociación, la red partidaria del PP constituye un sistema estable de relaciones que facilita la articulación de los distintos niveles de Administración y los intercambios políticos entre los actores implicados en el conflicto.
La Xunta nota los primeros síntomas de apaciguamiento a partir de la formación de un nuevo gobierno central. El presidente de la Xunta se beneficia del contexto de distribución de puestos políticos en el seno del PP. En efecto, la Administración pública española, tanto la central como la autonómica, se caracteriza por una fuerte politización de los cuadros de dirección. Los titulares de los puestos de director general y secretario general técnico cambian en función de las mayorías políticas (Ramió y SuBIRATS, 1996). Así pues, el nombramiento en Madrid de numerosos ministros y altos cargos procedentes de la Administración gallega situó al gobierno gallego durante varias semanas en una situación de cambio permanente. Esta situación excepcional dio la ocasión al Presidente de la Xunta de dar a una primera satisfacción a la coordinadora de afectados y a los alcaldes del PPdeG, con la destitución del Conselleiro de Educación, Piñeiro Permuy, quien pasó a ocupar la dirección de un organismo independiente, el Consejo Económico y Social, del Ministerio de Educación central, fue reemplazado por Celso Currás, próximo al presidente de la Diputación de Lugo y al secretario general del PPdeG, y favorable a una aplicación más flexible de la LOGSE. Su primera decisión fue destituir al equipo del ex-Conselleiro encargado de redactar la red de centros ESO.

A continuación, la Xunta entabló negociaciones a dos niveles distintos. A escala regional el nuevo Conselleiro de Educación comenzó las discusiones con los distintos grupos sociales y políticos movilizados, y a escala nacional solicitó la intervención del Ministerio de Educación. La transversalidad de las redes partidarias permitió la articulación de estas dos arenas de negociación. De este modo, en una primera tanda el Conselleiro de Educación negocia directamente con los alcaldes del Partido Popular y la coordinadora de afectados. Entonces, en el momento en que las protestas de numerosas comunidades rurales de España se alzan contra la ESO, la Xunta adopta una estrategia de nacionalización de las cuestiones en conflicto. Afirma que la solución al conflicto le corresponde al Ministerio de Educación. Las condiciones que posibilitan tal estrategia residen esencialmente en la existencia de la red partidista, a la que antes nos referimos, que permite contar a la Adminis. tración central con la existencia de un conjunto de interlocutores bien dispuestos a la negociación. Desde la formación del nuevo gobierno en Madrid, con el nombramiento de Esperanza Aguirre al frente de la cartera de Educación y Cultura, los parlamentarios regionales del PP de la provincia de Lugo indicaron a los alcaldes de los pequeños municipios rurales que la solución al problema de la ESO pasaría por la intervención de la Administración central. El objetivo de esta estrategia es, por una parte, disminuir la intensidad del conflicto a escala regional y, por otra, obtener compensaciones económicas para la aplicación de la LOGSE en Galicia. En efecto, dar satisfacción a los alcaldes y a la coordinadora de afectados supone aumentar el número de los centros autorizados a impartir los cursos de 
la ESO en zonas rurales, y dotarlos, en consecuencia, de los medios humanos y materiales precisos. Una actuación de este tipo colocaría en una situación financiera delicada a la Consellería de Educación. Así pues, la Xunta buscaría obtener la participación del Ministerio de Educación.

El desplazamiento del debate hacia la Administración central se tradujo en una disminución de las movilizaciones en el espacio regional. Los alcaldes del PPdeG y la coordinadora se reunieron a intervalos regulares con el Conselleiro de Educación y fueron progresivamente poniendo fin a sus actos de protesta. Paralelamente, a partir del mes de junio la Xunta comenzó sus negociaciones con el Ministerio de Educación. Desde el inicio de estas negociaciones el Conselleiro utilizó el registro de los grupos movilizados, subrayando las especificidades de la Galicia rural ${ }^{26}$. Sin embargo, la Xunta no consiguió que la Administración central dotase una financiación complementaria para el inicio del curso 1996-1997 destinada a la aplicación de la LOGSE en Galicia. El gobierno central financia en exclusiva la aplicación de dicha Ley en las Comunidades que no se beneficiaron del traspaso de las competencias en materia educativa y no desea financiar más allá de este ámbito. Por otra parte, los márgenes de discrecionalizar son extremadamente limitados, en la medida que el principal eslogan del gobierno Aznar era el de hacer entrar a España dentro de los criterios establecidos por el acuerdo de Maastricht.

Finalmente, la Xunta ratificó un compromiso muy favorable a los alcaldes y a la coordinadora de afectados, pero que se aleja de los objetivos iniciales de la LOGSE. La Consellería de Educación flexibilizó al máximo las modalidades de aplicación de esta Ley. El período transitorio de aplicación de la LOGSE se extendió a un número más elevado de alumnos de colegios públicos. El principio de indivisibilidad de los cursos de la ESO no fue aplicado y la Consellería aceptó igualmente la ampliación de la red de centros a numerosos colegios de la Galicia rural ${ }^{27}$. De este modo, en lugar del $68 \%$ previsto, el $82 \%$ de los que comienzan su primer curso de ESO permanecen en su colegio de origen frente al $64 \%$ de la media estatal. Estas medidas limitan a trescientos el número de alum. nos que deberán abandonar su comunidad para seguir los cursos de la ESO en lugar de los millares previstos al comienzo del conflicto. Esta forma partidista de regulación del conflicto de la ESO permitió resolver el conflicto político y dar satisfacción a los grupos movilizados, pero no fue capaz de resolver inmediatamente los problemas financieros y de organización de la enseñanza en Galicia.

De este modo, los sindicatos de profesores y el PSdeG se pronunciaron a finales del mes de julio contra el caos administrativo provocado por la actuación de la Xunta y el coste de las nuevas modalidades de aplicación de la ley. La reentrada del curso 1996-1997 se desarrolló en condiciones precarias con una ausencia indignante de personal educativo. En el momento de comenzar el curso numerosos alumnos no pudieron recibir ciertas materias optativas por falta de personal. Finalmente, a más largo plazo, la Xunta debe reunir los medios financieros necesarios para generalizar la aplicación prevista de la LOGSE en el plazo de cuatro años, en particular para la construcción de nuevos centros ESO. Las negociaciones transversales y los intercambios políticos en el marco del PP entre los grupos infrarregionales movilizados, la Xunta y el Ministerio de Educación nacional apaciguaron momentáneamente las movilizaciones en el espacio regional, pero no resolvieron las dificultades estructurales de aplicación de la LOGSE en Galicia.

\section{Conclusión}

Incluso si este estudio de caso no aporta más que elementos parciales de análisis, permite por lo menos medir ciertos efectos de la autonomía regional sobre los procesos de acción pública en España. Desde el punto de vista analítico, es esencial no homogeneizar el papel de los mesogobiernos en este proceso (Gomá y Subirats, 1998). Según los territorios y las configuraciones sociopolíticas, sus prácticas varían en gran medida. De entre todas las variables que deben ser tenidas en cuenta es importante destacar algunas de ellas como su Estatuto de Autonomía, su coyuntura política, la estructura de competición politica regional, la filiación partidista del gobierno autonómico y sus relaciones con el poder central. Estas variables orientan el posicionamiento de los actores en los procesos de acción pública y, por consiguiente, el de los mesogobiernos.

De este modo, en la Comunidad Autónoma de Galicia, una identidad regional preexistente aliada a una fuerte estructuración del espacio de competición político regional tiende a territorializar los asuntos políticos. La temática de la defensa de los intereses regionales se convirtió en el estímulo del debate político como blanco de la acción del gobierno autonómico. Este cambio en las percepciones de los problemas se manifiesta en los actores políticos y sociales en un fuerte potencial movilizador en la defensa de los intereses que ellos consideran propios de la Comunidad. Desde entonces, los conflictos de interés, las luchas por la definición de la realidad social entre los múl. tiples actores implicados se libran en el espacio político regional, en el centro del cual se halla el gobierno autonómico. Este estudio de caso nos muestra que estos fenómenos afectan a la actuación pública. Los grupos sociales y políticos cuya movilización se inscribió en la historia sociopolítica de Galicia, consiguieron a través de la intermediación de la organización partidaria del PPdeG forzar al gobierno autonómico a negociar los modos de aplicación de la LOGSE e ignorar algunos de sus principios. De este modo, la Comunidad Autónoma se constituyó en un foro y una arena a nivel regional de una política 
pública donde se discuten y negocian las normas y objetivos de una política previamente establecida a nivel central.

En el momento de la regulación del conflicto de la ESO la red partidista del PP es un elemento particularmente determinante. Suministra un marco de relaciones estable donde son posibles las transacciones y los intercambios políticos transversales. El conjunto de los actores y de los niveles de Administración que participan en la regulación del conflicto se inscriben dentro de esta red. Se puede aquí establecer la hipótesis de una fuerte politización de los procesos de acción pública en España. Muchos de los arbitrajes y de los compromisos políticos entre los diferentes actores y niveles de Administración parecen realizarse en el seno de las organizaciones partidistas. Para progresar más todavía en el análisis sería necesario realizar estudios comparados entra las distintas Comunidades Autónomas con la finalidad de comprender, según los territorios, el funcionamiento concreto de los mesogobiernos y su articulación con la Administración central.
Traducción de Miguel Anxo Bastos Boubeta.

' Nuestro trabajo de tesis, en el que este estudio se inscribe, pretende realizar un estudio comparado Francia/España sobre la capacidad política de las instituciones regionales y la estructuración de intereses en el ámbito de la ordenación del territorio. Escogimos cuatro territorios de investigación, dos regiones francesas, Bretaña y Centro, y dos Comunidades Autónomas españolas, Galicia y La Rioja.

* Centre de Recherches Administratives et Politiques. Université Rennes I.

${ }^{2}$ Con el fin de analizar en exclusiva las dinámicas de conflicto y regulación durante el período de aplicación de la LOGSE centramos nuestro análisis en el periodo de marzo a septiembre de 1996, eludiendo deliberadamente el estudio de las actuaciones públicas llevadas a cabo después de este período por la Consellería de Educación de la Xunta.

${ }^{3}$ En 1981 la tasa de abstención en las elecciones autonómicas fue del $53,7 \%$ frente al $34,7 \%$ en 1993 .

${ }^{4}$ Nacidos en la región que representan los diputados regionales son más jóvenes que los electos en elecciones locales. Su nivel de estudios es elevado y su situación profesional muestra que son fruto de los cambios socioeconómicos que ha conocido España en los años sesenta. Por otra parte, las trayectorias de los diputados autonómicos muestra que se caracterizan por una fuerte militancia partidista a diferencia de los electos a escala local (GENIEYs, 1996).

3 Los partidos de derecha o de centro-derecha han ganado todas las elecciones autonómicas en Galicia (1981, 1985, 1989, 1993, 1997). Son igualmente mayoritarios en esta región en todas las elecciones generales celebradas después de la transición democrática (VILAS Nogueira, 1992). Es más, Galicia fue durante mucho tiempo la única de las grandes Comunidades Autónomas controladas por la derecha española. Por otra parte, los dirigentes del Partido Popular, especialmente Aznar y Fraga, han presentado a Galicia como un escaparate de la política que quisieran desarrollar en el conjunto de España.

' José Manuel Romay, presidente del PPdeG de la provincia de A Coruña, miem. bro de la Xunta, se convirtió en Ministro de Sanidad en tanto que Mariano Rajoy, también miembro del gobierno autonómico, obtiene el Ministerio de Administraciones Públicas (en la reorganización gubernamental de enero de 1999 le fue asignada la cartera de Educación y Cultura). A estos dos hombres hay que añadir los numerosos directores generales de la Administración autonómica, principalmente de la Consellería de Economía, que accedieron a altos cargos en la Administración central.

${ }^{7}$ El reagrupamiento de varios partidos nacionalistas en el seno del Bloque Nacionalista Galego (BNG) se tradujo en una sensible progresión del voto nacionalista en Galicia. En las elecciones autonómicas de 1993 el BNG obruvo el. 18,5\% de los votos y 13 diputados en el Parlamento autonómico. Esta evolución se vio refrendada con la conquista, histórica, de dos escaños en las Cortes en las elecciones de marzo de 1996. En las elecciones de 1997 consiguió convertirse en la segunda fuerza política de Galicia al obtener cerca del $25 \%$ de los votos ( 400.000 votos aproximadamente) y 18 escaños en el Parlamento gallego.

${ }^{8}$ La dimensión estratégica del registro regionalista es importante. De este modo, el discurso «galleguista» del PSdeG.PSOE se acentuó, un año después de la derrota socialista en las elecciones generales, con la proximidad de las elecciones autonómicas gallegas de 1997 .

${ }^{9}$ La ESO va a poner fin progresivamente (en el curso 2000-2001) al sistema anterior que dividía la escolaridad en tres fases: EGB (Enseñanza General Básica) obligatoria hasta los catorce años; BUP (bachillerato) y COU (curso de orientación universitaria). Precisemos, en fin, que la Ley establece que la ESO se divida en dos fases de dos años cada una y que deberán ser impartidos de forma indivisible en un mismo centro. Este último aspecto es importante en la medida en que, para salir del conflicto, la Xunta flexibilizará al máximo la aplicación de este principio.

${ }^{10}$ El esquema organizativo de la Administración autonómica en Galicia está inspirado en los modelos vasco y catalán, ellos mismos fuertemente influenciados por el modelo burocrático de la Administración Central del Estado (Ramio y SuBIRATS, 1996). De este modo, la Xunta se divide en diez departamentos con un Conselleiro a su cabeza, el cual desempeña funciones de ministro en el gabinete regional. Por otra parte, parece que las reformas administrativas puestas en práctica en los años 80 en ciertas Comunidades Autónomas, especialmente en Galicia, buscaron más la racionalización de los organismos administrativos que construir modelos regionales de administración (VLLAS Nogueira y BASTOS BoubETA, 1997).

"Fuertemente endeudadas, los márgenes de maniobra financieros de las Comunidades Autónomas continúan siendo muy estrictos. El Estado central conserva el control sobre los recursos. Las Comunidades Autónomas que cuentan con un elevado nivel de autonomía están siempre procurando obtener nuevos recursos fiscales para hacer frente a las exigencias derivadas de las competencias que ejercen. En 1997 la Xunta estableció un presupuesto superior en recursos gracias a los flujos financieros de la Unión Europea.

${ }^{12}$ Galicia padece desequilibrios socioeconómicos de importancia. En efecto, se da una verdadera segmentación territorial entre la franja costera, poblada y económicamente desarrollada (abarca $2 / 3$ de la población gallega concentrada principalmente en la provincia de A Coruña y parte de la de Pontevedra) y la zona interior, eminentemente rural, que padece un fuerte proceso de despoblación (provincias de Lugo y Ourense). De esta forma, los efectos previsibles de la racionalización de los centros ESO son particularmente negativos en las zonas desfavorecidas de la Galicia interior, donde la desertificación amenaza a numerosas comunidades.

${ }^{13}$ Las primeras manifestaciones tuvieron lugar el 15 de marzo de 1996 en la provincia de Lugo (varios centeneres de manifestantes) y fueron especialmente dispersas. La mayor parte de los habitantes de los municipios afectados no se desplazó a la capital provincial y se manifestó en sus propios lugares de residencia. Las asociaciones de padres de alumnos y de alcaldes que se desplazaron a la capital provincial se agruparon por municipios y realizaron sus demandas delante de la autoridad de forma individualizada.

${ }^{14}$ Galicia cuenta con 315 municipios y 3.773 parroquias. Los municipios gallegos son mucho más grandes que en el resto de España y se componen de numerosas entidades de población. En promedio, cada municipio agrupa 12 parroquias de una superficie de $7,7 \mathrm{~km}^{2}$ (Fusl, 1989). Por otra parte, cada parroquia engloba múltiples lugares de población dispersos en el campo. 
15 El caciquismo encubre el conjunto de mediaciones clientelares que permitieron, bajo el régimen de la Restauración (1875-1923), la alternancia en el poder de los partidos liberal y conservador (turno) gracias a elecciones amañadas y al control de los elcctores por los gobernadores civiles y los caciques locales (KERN, 1973).

${ }^{16}$ Los partidos políticos débilmente implantados en las zonas rurales de Galicia como el BNG o el PSdeG denuncian sistemáticamente el caciquismo y el clien. telismo del PPdeG.

17 G. Mhrquez Cruz (1995) muestra que en las primeras elecciones locales en 1979, cl 33\% de los alcaldes electos en Galicia ya lo eran en 1973 (en esta fecha los alcaldes eran designados por el Régimen) frente al 14,2\% en Andalucía. En las elecciones de 1995 el 10,8\% de los alcaldes electos en Galicia ocupaban la misma función en 1973 y 1979. Si se considera el número total de electos locales (concejales) el porcentaje se eleva hasta el 19,8\%. Esta continuidad de las élites locales concierne especialmente al PPdeG, ya que el $14,4 \%$ de los alcaldes elegidos bajo dichas siglas ya ocupaban este puesto en 1973 frente al 1,8\% de los electos bajo las siglas del PSdeG.PSOE.

${ }^{18}$ Al legislar sobre el sistema político local los gobiernos autonómicos tratan de limitar o de organizar de forma distinta los poderes de los municipios y las diputaciones provinciales que se encuentran en su territorio. Pero la legislación varía mucho según las regiones y las reglas de juego políticas se modifican atendiendo a las relaciones entre las Comunidades Autónomas y las Administraciones locales. Así, en lo concerniente a la puesta en práctica de los proyectos LEADER en Extremadura, A. SMITH apunta que el gobierno autonómico ha debido imponer sus objetivos frente a los dos representantes de las Diputaciones provinciales.

${ }^{19}$ En las otras provincias la tasa de afliiación al PPdeG de los alcaldes son también significativas: $78,3 \%$ en Ourense, $70,5 \%$ en Pontevedra y $61,7 \%$ en A Coruna (MARQuez Cruz, 1995).
${ }^{20}$ Todas estas afirmaciones fueron extraídas de reportajes del conflicto aparecidos en La Voz de Galicia y El Correo Gallego de las fechas en que se desarrollaron las movilizaciones.

${ }^{21}$ No es su posicionamiento político la que la distingue, sino la naturaleza de sus componentes. La Federeración de Municipios Gallega agrupa esencialmente a los municipios rurales.

${ }^{22}$ No detallamos aquí las demandas más concretas de la coordinadora, como, por ejemplo, el mantenimiento en los colegios rurales de servicios de comedor o de transporte para los alumnos.

${ }^{23}$ Puede considerarse que los principales partidarios de la reforma son los representantes de la enseñanza privada en Galicia. En efecto, el reagrupamiento de los centros puede permitir esperar un flujo de alumnos hacia los colegios privados, particularmente en las zonas rurales.

${ }^{24}$ Por ejemplo, en la provincia de A Coruña la relación entre diputado y número de electores es de 1 a 36.800 , mientras que en la provincia de Lugo es de 1 a 22.350 (VILAS Nogueira, 1992).

${ }^{25}$ A. SMITH (1995) subraya igualmente la dimensión transversal del sistema político español. Insistimos aquí sobre la capacidad de esta red partidista de articular diferentes niveles de administración y de constituir un marco de negociación para los diversos actores políticos implicados en el conflicto.

${ }^{26}$ Después de su primera entrevista con la Ministra de Educación, Celso CurRás declaró que la implantación de la LOGSE en Galicia plantea graves problemas ligados a las características del territorio y especialmente a la existencia de una multitud de aldeas rurales y semiurbanas (25 de junio de 1996, La Voz de Galicia).

${ }^{27}$ Después del anuncio de esta extensión del plazo de aplicación, a mediados de julio de 1996, se asiste a manifestaciones de júbilo en los municipios afectados, en especial en la provincia de Lugo.

\section{Bibliografía}

AcrunoFf, R. (1993), «Las relaciones intergubernamentales y el Estado de las Autonomías», en Politica y Sociedad, No. 13, pp. 87-105.

Botrela, J. (1989), «The Spanish New Regions: Territorial and Political Pluralism», en Intermational Political Science Review, vol. 10, No. 3, pp. 263-271.

BrıQuet, J. L., y SAwICK, F. (1989), «L'analyse localisée du politique. Lieux de recherche ou recherche de lieux», en Politix, No. 7/8, pp. 1-11.

Cazorla Pérez, J. (1994), «El clienteismo de partido en España ante la opinión pública. El medio rural, la Administración y las empresas», Working Papers, No. 86, 1994.

DE Miguel, A.; y LinZ, J. J. (1996), «Within-nation differences and comparisons: the Eight Spain», en R. L. MerRIT y S. RoKKan (1966), Comparing Nations, New Haven: Yale University Press, pp. 267-320.

Dinz Lopez, C. E. (1982), «The Politization of Galician Cleavages», en S. Rokxan y D. UnwIN (1982), The Politics of Territorial Identity. Studies in European Regionalism, London: Sage, pp. 389.424.

Fusı, J. P. (1989), «Galicia», en J. P. Fusı (dir.), España: Autonomias, tomo V, Madrid: Espasn-Calpe, pp. 467.515.

Garraud, P. (1994), «Le processus «autonomique» et la fin de l'exception espagnole", en R. Balme, P. Garraud, V. Hoffmann-Martinot y E. Rttaine, Le temtoire pour politiques: variations européennes, L'Harmattan, pp. 161-203.

GinIEYS, W. (1996), «Las élites péripheriques espagnoles face au changements de régime», en Revue Française de Science Politique, vol. 46, No. 4, aout, pp. 650.680 .

Gomi, R., y Subirnts, J. (coords.) (1998), Políticas públicas en España, Barcelona: Ariel.
Gunther, R.; SANI, G., y SHABAD, G. (1988), Spain after Franco: The Making of a Competitive Party System, University of California Press.

KeRn, R. W. (1973), «The Spanish Caciquismo, a Classic Model», en Kern, R. M. (ed.), The Caciques. Oligarchical Politics and the System of Caciquismo in the Luso-Hispanic World, New Mexico: University of New Mexico Press, pp. 42.55.

ItURRA, R. (1988), Antropología económica de la Galicia rural, Santiago: Xunta de Galicia.

Linz, J. J. (1973), «Early State-Building and Late Peripherical Nationalism Against the State», en S. N. EISENSTADT (ed.), Building States and Nations, London: Sage, vol. 2, pp. 32-116.

Lisón Tolosana, C. (1981), Antropología cultural de Galicia, Madrid: Siglo XXI.

Málz, R. (1996), «Nación de Breogán: Oportunidades políticas y estrategias enmarcados en el nacionalismo gallego 1886-1996», en Revista de Estudios Politicos, No. 92, abril-junio, pp. 33-75.

Márquez CRUz, G. (1995), «El gobierno local en Galicia: Resultados electorales, elites políticas locales y producción de gobierno (1979-1995)», FEGAMP, No. 10, Outono.

Morata, F. (1993), «L'État des autonomies un modèle hybride», en Dossiers et Débats. L'administration territoriale en Europe, pp. 37.55.

Pérez Diaz, V. (1994), La primacia de la sociedad civil, Madrid: Alianza.

Romero-Maura, J. (1977), «Caciquismo as a Political System», en E. Gellner, y Watterbury, A. J. (1977), Patrons and Clients in Mediterranean Societies, London: Ducworth, pp. 53.62.

SAwICK, F. (1988), «Questions de recherche: pour une analyse locale des partis politiques», en Politix, No. 2, pp. 2.17. 
SMrTh, A. (1995), «Le local, l'État et la Commission face aux comanautés autonomes. L'exemple de la mise en place du programme LEADER en Espagne», en Ple Sud, No. 3, Automne, pp. 103-116.

SMrTH, A. (1997), «L'echalon infraregional: lieu privilégié des fonds structurels», en P. Le Galis y C. Lequesne (dirs.), Les paradoxes des régiones en Europe, París: La Découverte, pp. 57-71.

SubiRaTs, J. (1991), «Un problema de estilo. La formación de políticas públicas en España», Cuadernos y Debates, No. 38, Madrid: Centro de Estudios Cons. titucionales.
VALıÉs, J. M., y CuCHшO, M. (1988), «Decentralisation in Spain: A Review», en European Joumal of Political Research, vol. 16, No. 4, pp. 395-407.

Vilas Nogueira, J. (1992), «Las elecciones en Galicia (1976-1991)», Working Papers, No. 57, Barcelona.

Vilas Nogueira, J., y Bastos Boubeta, M. A. (1997), «Reforma y modernización administrativas. Especial referencia a la Comunidad Autónoma de Galicia», en Gestión y análisis de politicas públicas, No. 7-8, septiembre 1996-abril 1997, pp. 165-175.

Vilas Nogueira, J., et al. (1994), O Sistema Político Galego, Vigo: Xerais. 